Service social

\title{
Dépistage systématique de la violence conjugale. Réflexion théorique et développement d'un protocole
}

\section{Maryse Rinfret-Raynor et Joane Turgeon}

Volume 44, numéro 2, 1995

Visages de la violence

URI : https://id.erudit.org/iderudit/706693ar

DOI : https://doi.org/10.7202/706693ar

Aller au sommaire du numéro

Éditeur(s)

École de service social de l'Université Laval

ISSN

1708-1734 (numérique)

Découvrir la revue

Citer cet article

Rinfret-Raynor, M. \& Turgeon, J. (1995). Dépistage systématique de la violence conjugale. Réflexion théorique et développement d'un protocole. Service social, 44(2), 57-90. https://doi.org/10.7202/706693ar
Résumé de l'article

À la suite d'une démarche de planification socio sanitaire visant à définir les priorités d'action pour les années à venir, la violence conjugale est devenue une des deux problématiques prioritaires pour le CLSC Saint-Hubert et son dépistage, un objectif important du plan opérationnel. Pour atteindre cet objectif, le CLSC a fait appel au Centre de recherche interdisciplinaire sur la violence familiale et la violence faite aux femmes (CRI-VIFF). Une équipe de recherche de ce centre a travaillé à l'élaboration d'un protocole de dépistage systématique de la violence conjugale. Cet article d écrit l'élaboration de ce protocole et son application par un nombre limité d'intervenants et d'intervenantes auprès d'une partie de la clientèle du CLSC. Cette phase expérimentale précède l'application généralisée du protocole dans tous les services du CLSC 


\title{
Dépistage systématique de la violence conjugale Réflexion théorique et développement d'un protocole
}

\author{
Maryse RINFRET-RAYNOR \\ Professeure titulaire \\ École de service social, Université de Montréal \\ Joane TURGEON \\ Psychologue et chercheure \\ École de service social, Université de Montréal
}

\section{CONTEXTE DE LA RECHERCHE}

En octobre 1991, le CLSC Saint-Hubert entreprend une démarche de planification sociosanitaire en vue $d^{\prime}$ identifier certains problèmes présents sur son territoire et de déterminer lesquels deviendront les cibles prioritaires d'action pour les années à venir. Cette démarche comprend une consultation de son personnel et des membres de la communauté de Saint-Hubert, en plus d'une étude approfondie des données sociosanitaires et sociodémographiques du territoire. Au terme de ce processus, la violence conjugale devient une des deux problématiques prioritaires pour le CLSC. 
Un comité multidisciplinaire est alors mandaté pour déterminer la perspective à adopter et les actions à entreprendre eu égard à cette problématique et pour définir les objectifs-cadres, c'est-à-dire les objectifs généraux qui encadreront les actions du plan opérationnel. Le premier de ces objectifs s'énonce ainsi: Dépister la violence conjugale chez la clientèle qui utilise nos services.

Consciente des difficultés du dépistage, l'équipe du CLSC Saint-Hubert choisit de s'associer au Centre de recherche interdisciplinaire sur la violence familiale et la violence faite aux femmes (CRI-VIFF) pour élaborer un protocole de dépistage systématique de la violence conjugale. Le présent article décrit ce protocole et son application expérimentale par un nombre limité $d^{\prime}$ intervenants et $d^{\prime}$ intervenantes auprès d'une partie de la clientèle du CLSC ${ }^{1}$. Cette phase précède I'application généralisée du protocole dans tous les services du CLSC.

\section{Clarification de la notion de dépistage}

L'équipe de recherche s'est entendue pour définir le dépistage comme un processus actif $d^{\prime}$ intervention permettant de découvrir la présence de certains problèmes (présents ou en devenir) par la recherche systématique de signes plus ou moins apparents. Le dépistage constitue donc une forme $d^{\prime}$ intervention préventive.

La prévention comprend toutes les formes d'intervention visant à empêcher l'apparition de certains problèmes ou à limiter leur évolution. Pour atteindre ces objectifs, il est nécessaire de connaître les signes avant-coureurs de leur apparition et les mécanismes de leur développement.

Trois types de prévention, liés au moment de l'action préventive, ont été mis en évidence: la prévention primaire, secondaire et tertiaire. Le dépistage permet $d^{\prime}$ intervenir aux trois moments de prévention.

La prévention primaire comprend toutes les activités qui sont réalisées pour prévenir différents problèmes et protéger les états de santé (Bloom, 1987). À ce niveau de prévention, les efforts sont réellement proactifs: on agit avant que le problème ne se présente. La prévention primaire tente d'abaisser la prévalence de cas existants en réduisant la fréquence future, c'est-à-dire le taux d'apparition des

1. Nous tenons à remercier Mme Lucie Joyal, ainsi que toutes les personnes du CLSC Saint-Hubert ayant participé à cette recherche. Sans leur contribution, la réalisation de ce projet aurait été impossible. 
nouveaux cas. Son action est orientée vers des personnes pouvant être à risque à cause de leur situation de vie.

Toutes les femmes sont visées par la prévention primaire de la violence conjugale. Les stratégies de prévention primaire sont axées sur les facteurs de protection: fournir aux femmes de l'information sur la violence conjugale et les sensibiliser aux processus en cause dans l'escalade; les aider à reconnaître et à nommer la violence lorsqu'elle se présente et à mettre en œuvre des moyens pour la contrer (Turgeon, 1992). Le dépistage de la violence conjugale permet $d^{\prime}$ identifier les femmes présentant certains facteurs de risque dans le but d'éviter que la violence n'éclate dans leur vie.

La prévention secondaire, qu'on appelle aussi intervention précoce, consiste à intervenir le plus tôt possible, aux premiers signes des problèmes (Pransky, 1991). Elle vise à réduire le taux de prévalence des problèmes en réduisant leur durée chez les personnes qui les subissent.

Le but de la prévention secondaire de la violence conjugale est d'intervenir efficacement aussitôt que des signes précurseurs de la violence se manifestent (Turgeon, 1992). Le dépistage permet une intervention précoce auprès des femmes qui, par certains signes, laissent entrevoir qu'une situation de violence conjugale s'installe dans leur vie.

La prévention tertiaire ne constitue pas de la prévention au sens strict du terme, puisque son objectif est d'éviter que les personnes qui ont déjà éprouvé un problème particulier le revivent une autre fois (Pransky, 1991). Elle comprend toutes les activités destinées à réduire au minimum les difficultés de fonctionnement consécutives à ce problème.

La prévention tertiaire de la violence conjugale correspond à I'intervention qui suit le plus rapidement possible les manifestations de la violence (Turgeon, 1992). Le travail avec les victimes leur permet de surmonter les difficultés découlant de la violence qu'elles ont subie (Conférence nationale sur la violence familiale, 1989). Le dépistage permet de reconnaître aussitôt que possible qu'une cliente est victime de la violence de son partenaire et de lui apporter rapidement un soutien réel pour mettre fin à cette situation ou prévenir sa réapparition (Brekke, 1987 ; McCaughry et Cameron, 1994).

Il importe de rappeler que le but du dépistage n'est pas de faire prendre conscience aux victimes qu'elles sont violentées. L'objectif $d^{\prime}$ aider les femmes violentées à reconnaître et à nommer leur victimisation peut faire partie d'un éventuel plan d'intervention. Cependant, au moment du dépistage, il est essentiel que l'intervenant ou 
I'intervenante sache que sa cliente subit une situation de violence qui affecte sa qualité de vie et sa santé. L'intervention sera plus efficace en tenant compte de cette réalité, peu importe que la femme reconnaisse ou non ce qu'elle subit comme étant de la violence conjugale.

Que I'on travaille dans le champ de la médecine, des sciences infirmières, de la psychologie ou du travail social, le dépistage de la violence conjugale est une intervention préventive essentielle: $c^{\prime}$ est le premier pas qui permet de répondre efficacement aux besoins des victimes en tenant compte de leur situation réelle et de leurs besoins particuliers.

\section{Conséquences de la violence conjugale}

En novembre 1993, I'enquête de Statistique Canada sur la violence envers les femmes (Statistique Canada, 1993) arrive à la conclusion suivante: une femme sur trois subit, au cours de sa vie, de la violence de la part d'un partenaire ou ex-partenaire. Un problème d'une telle ampleur mérite qu'on le dépiste, d'autant plus que ses conséquences multiples affectent la santé physique et mentale des femmes violentées. Étant donné que dans cette étude la violence conjugale sera dépistée par la voie de ses conséquences, celles-ci sont brièvement présentées.

La violence conjugale comporte des effets néfastes sur l'intégrité physique des femmes qui la subissent. Celles-ci présentent divers types de blessures (Hodgins et Larouche, 1982; OIIQ, 1987; Statistique Canada, 1993), dont certaines sont parfois très graves: blessures aux organes internes (Ghent, Da Sylva, Farren, 1982; Rodgers, 1994), à la tête, au visage, à l'abdomen (Rinfret-Raynor et al., 1989), fractures du nez, de la mâchoire (Ghent, Da Sylva, Farren, 1982), etc. Sans oublier qu'à l'extrême limite de l'escalade de la violence certaines femmes se font tuer par leur partenaire.

D'autres conséquences physiques de la violence sont moins directes, mais non moins réelles: I'état de santé général des femmes violentées est moins bon que celui des femmes dont la vie de couple est exempte de violence (Chénard, Cadrin, Loiselle, 1990; Follingstad et al., 1991 ; Kérouac, Taggart et Lescop, 1986). Par exemple, les femmes violentées souffrent de perte d'appétit, de maux de tête, de crampes d'estomac et d'ulcères (Follingstad et al., 1991; Lacombe, 1990; OIIQ, 1987). On retrouve chez les femmes violentées beaucoup plus que chez les autres femmes des cas d'insomnie, de fatigue générale et d'anémie (Ghent, Da Sylva, Farren, 1982; Kérouac et al., 1986 ; Larouche, 1985 ; OllQ, 1987). 
Les conséquences psychologiques de la violence conjugale sur les femmes sont aussi très nombreuses et de plus en plus souvent associées dans la littérature à un état de stress post-traumatique (Dutton et Goodman, 1994; Gleason, 1993; Houskamp et Foy, 1991; Kemp, Rawlings et Green, 1991).

Turgeon (1995) a d'ailleurs démontré qu'il est possible de regrouper la majorité des symptômes psychologiques relevés dans plusieurs études sur les femmes violentées en fonction des six critères diagnostiques de l'état de stress post-traumatique tel que décrit dans le Diagnostic and Statistical Manual of Mental Disorders (DSM-IV) de I'American Psychiatric Association (1994). Ces critères sont les suivants :

A. La personne a été exposée à un événement traumatique au cours duquel (1) elle a vécu ou a été témoin d'un ou de plusieurs événements impliquant une mort, une menace de mort, une blessure sérieuse ou une menace de blessure sérieuse ou une menace à son intégrité physique ou à celle d'autrui et (2) elle a vécu des sentiments de peur intense, d'impuissance ou d'horreur.

II a déjà été mentionné que les femmes violentées subissent divers types de blessures. Elles sont fréquemment exposées à des situations de danger pour leur vie, leur intégrité physique ou celle de leurs enfants. Elles éprouvent des sentiments d'impuissance (Walker, 1979, 1984; Walus Wigle et Meloy, 1988) ou de peur intense (Gianakos et Wagner, 1987 ; Kemp, Rawlings et Green, 1991 ; Statistique Canada, 1993; Walker, 1985).

B. L'événement traumatique est revécu de différentes façons.

Les femmes violentées revivent dans leur esprit les situations de violence qu'elles ont subies. Des souvenirs de ces événements s'imposent à elles de façon envahissante (Kemp, Rawlings et Green, 1991). Ils prennent parfois la forme d'images, de cauchemars ou de rêves répétitifs (Hilberman et Munson, 1977; Walker, 1984, 1985), provoquant des sentiments de détresse ou de terreur (Hilberman et Munson, 1977). Des femmes violentées vivent des sentiments de détresse lorsqu'elles sont exposées à des événements qui leur rappellent la violence subie (Kemp, Rawlings et Green, 1991).

C. Les stimuli associés au traumatisme sont évités ou la réactivité générale est émoussée.

Les femmes violentées ont des réactions d'évitement et de déni (Kemp, Rawlings et Green, 1991 ; Turner et Shapiro, 1986). Elles peuvent éviter de penser aux événements subis, avoir de 
la difficulté à se rappeler certains aspects de ces événements, éviter les stimuli qui leur rappellent la violence (Dutton et Painter, 1993; Hilberman et Munson, 1977 ; Walker, 1985). Elles ont parfois le sentiment que l'avenir est noir (Rodgers, 1994 ; Walker, 1984). Certaines femmes en viennent à considérer que la mort est la seule issue (Chénard, Cadrin, Loiselle, 1990 ; Kérouac et al., 1986).

D. La personne présente des symptômes traduisant une hyperactivité neurovégétative.

L'augmentation de I'activation physiologique se manifeste de différentes façons chez les femmes violentées: problèmes de sommeil (Dutton et Painter, 1993; Kemp, Rawlings et Green, 1991; Walker, 1985), irritabilité (Adshead, 1994; Chénard, Cadrin et Loiselle, 1990; Rosewater, 1985), difficultés de concentration (Kemp, Rawlings et Green, 1991), hypervigilance (Hilberman et Munson, 1977 ; Kemp, Rawlings et Green, 1991 ; Walker, 1985), sursauts (Kemp, Rawlings et Green, 1991; Larouche, 1987). Elles souffrent aussi d'anxiété (Dutton et Painter, 1993 ; Follingstad et al., 1991; Hilberman et Munson, 1977 ; Rodgers, 1994 ; Walker, 1985), de nervosité (Chénard, Cadrin et Loiselle, 1990 ; Rinfret-Raynor et al., 1991) et de stress extrême (Frankel-Howard, 1989; Kérouac et al., 1986; Lacombe, 1990 ; Walker, 1984).

E. La durée de l'état de perturbation (relié aux symptômes des points $B, C$ et $D$ ) dépasse un mois, ce qui a été observé chez plusieurs femmes violentées (Dutton et Painter, 1993; Rosewater, 1985 ; Walker, 1984).

F. L'état de perturbation cause de la détresse ou des difficultés dans les relations sociales, au travail ou dans les autres domaines de la vie, ce qui a été observé chez plusieurs femmes violentées par Dutton et Painter (1993) et Rosewater (1985).

Comme les personnes qui vivent une situation de danger pour leur vie (victimes de viol, de vols à main armée, de catastrophes naturelles, vétérans de la guerre, etc.), plusieurs femmes violentées présentent ainsi des symptômes d'un état de stress post-traumatique.

Contrairement à d'autres diagnostics, l'état de stress posttraumatique reconnaît l'existence d'un événement traumatisant ayant un impact sur la personne qui le subit. Selon Dutton et Goodman (1994), l'état de stress post-traumatique est en fait formé d'un ensemble de réactions normales à un ou à des événements anormaux. L'identification de cet état chez des femmes violentées permet 
de conceptualiser la violence conjugale sans jeter le blâme sur les victimes (Houskamp et Foy, 1991). Elle permet aussi de souligner les conséquences néfastes de la violence sur la santé mentale des victimes et l'importance de mettre en œuvre des moyens concrets pour les protéger.

Même si les réactions des femmes violentées présentent des similarités avec celles des victimes d'autres types d'événements traumatiques, la comparaison avec ces phénomènes s'arrête là, car la violence conjugale demeure une problématique particulière à plusieurs égards. En effet, cette violence s'inscrit dans une relation amoureuse, elle se produit de façon répétée sur de longues périodes, elle évolue souvent à travers un cycle comportant une phase de "rémission» au cours de laquelle le partenaire présente des excuses et fait des promesses, et elle s'installe graduellement, souvent de façon très subtile, dans une escalade qui peut se rendre jusqu'au meurtre. Dans ce contexte, la violence conjugale n'a pas besoin d'être physique pour engendrer des conséquences extrêmement néfastes sur ses victimes. Dans leur étude, Kemp et al. (1995) ont constaté que $81 \%$ des femmes violentées physiquement ainsi que $63 \%$ des femmes violentées verbalement satisfaisaient aux critères d'un état de stress post-traumatique.

La violence conjugale a d'autres conséquences sur les femmes qui en sont victimes: leur estime d'elles-mêmes et leur confiance en elles sont très ébranlées (Aguilar et Nightingale, 1994; Dutton et Painter, 1993 ; Rodgers, 1994 ; Walker, 1979, 1983; Walus Wigle et Meloy, 1988). Plusieurs d'entre elles sont déprimées (Chénard, Cadrin, Loiselle, 1990 ; Dutton et Painter, 1993; Follingstad et al., 1991 ; Hilberman et Munson, 1977 ; Kemp, Rawlings et Green, 1991 ; Regroupement des CLSC du Montréal métropolitain, 1990 ; Rodgers, 1994; Walker, 1984) et certaines font des tentatives de suicide (Chénard, Cadrin, Loiselle, 1990).

\section{Difficultés de dépister la violence conjugale}

On sait que les femmes violentées consultent beaucoup les ressources formelles et informelles (Rinfret-Raynor et al., 1991). Pourtant, la situation de violence est rarement reconnue par les personnes consultées. Ainsi, Cantin, Rinfret-Raynor et Fortin (1994) ont fait l'analyse de 177 dossiers de femmes agressées par leur conjoint et dirigées vers les services de neuf CLSC de la région du Montréal métropolitain par les policiers et policières qui étaient intervenus à la suite $d^{\prime} u n$ appel d'urgence de la femme elle-même ou de quelqu'un du voisinage. 
Ces chercheuses ont constaté que le tiers des femmes de leur échantillon ( $n=58)$ avaient déjà consulté le CLSC où elles furent dirigées. L'existence de la violence conjugale $\mathrm{n}^{\prime}$ avait pourtant été reconnue que pour 12 d'entre elles.

La violence conjugale est très difficile à dépister et passe souvent inaperçue. Plusieurs facteurs contribuent à cette situation.

\section{Valeurs sociales}

La violence est une dimension de la vie familiale qui a été longtemps ignorée. Jusqu'à tout récemment, la violence conjugale était considérée comme un phénomène privé faisant partie des rapports entre certains adultes (Gauthier, 1991). La violence concernait donc exclusivement les deux personnes en cause (Lafrance, 1984). Même si, depuis quelques années, les normes sociales à l'égard de la violence conjugale se sont transformées, plusieurs personnes pensent encore qu'il est incorrect d'intervenir dans des «querelles de ménage » ou des «chicanes de famille»(Gendron, 1991). La violence conjugale demeure un sujet tabou pour les victimes comme pour les personnes qui en sont témoins, ce qui ne facilite pas son dépistage.

Les spécialistes du monde médical ou des services sociaux ne sont pas à l'abri de ces tabous. Pour respecter le caractère privé du mariage et de la famille, plusieurs se montrent réticents à s'impliquer dans les cas de violence conjugale (Johnson, 1985 ; MacLeod, 1980 ; Raymond et Charbonneau, 1984).

\section{Silence des victimes}

Dans ce contexte social, il est peu aisé pour les femmes violentées de dévoiler la violence qu'elles subissent. De plus, la menace de violence étant toujours présente, ces femmes vivent dans la peur de leur partenaire. Elles craignent des représailles, pour elle ou pour les enfants, s'il apprend qu'elles ont dévoilé la situation (Gendron, 1991 ; Lafrance, 1984; MacLeod, 1987). L'emprise psychologique du partenaire violent est aussi très forte, et peut amener certaines femmes à croire, comme leur partenaire l'affirme, qu'elles provoquent sa violence. Elles finissent par se sentir responsables de la situation (Campbell, 1989) : "Si je faisais mieux la cuisine, si j'étais plus ceci ou moins cela, ça n'arriverait pas.»

Des femmes nourrissent l'espoir que la situation s'améliore et que leur conjoint change (Gendron, 1991 ; Regroupement provincial des maisons $d^{\prime}$ hébergement et de transition pour femmes victimes de violence conjugale, 1990). Certaines ont honte de la violence qu'elles 
subissent, se sentent diminuées et dévalorisées (Gendron, 1987, 1991 ; Rinfret-Raynor et al., 1991 ; Rodgers, 1994). Elles hésitent à se confier par peur d'être jugées (Lafrance, 1984), de ne pas bénéficier d'une écoute sympathique (Gendron, 1991; MacLeod, 1987) ou d'être poussées à quitter leur conjoint (Carrier et Michaud, 1982). Ces peurs ne sont pas irrationnelles: malheureusement, plusieurs femmes violentées subissent encore des attitudes d'incompréhension de la part de personnes mal informées.

D'autres craintes peuvent inciter les femmes à se taire: la peur de briser la famille, la peur de perturber les enfants, la peur des réactions de la famille élargie, etc. Les femmes violentées consultent donc très rarement en spécifiant le problème de violence conjugale. Pour contrecarrer ce fait, les personnes de toutes les disciplines doivent acquérir une conscience aiguë de la dynamique et des signes de la violence.

\section{Non-spécificité des signes liés à la violence conjugale}

Comme il a été mentionné précédemment, les conséquences de la violence conjugale laissent des traces, parfois physiques et souvent psychologiques, chez les femmes qui en sont victimes. Cependant, plusieurs de ces signes ne sont pas propres à la violence conjugale et peuvent être associés à d'autres types de problèmes.

Il se révèle donc difficile pour les spécialistes de la santé mentale, comme les personnes intervenant en santé ou en sciences infirmières, de distinguer les symptômes des femmes victimes de violence de ceux des femmes souffrant de problèmes de santé mentale (Hilberman et Munson, 1977 ; Rosewater, 1985 ; Stark et al., 1979, 1981), avec pour conséquence qu'un faible pourcentage de femmes violentées font l'objet d'un diagnostic juste et de soins appropriés. En 1981, Stark et al. estimaient cette proportion à moins d'une femme violentée sur 25, et la situation ne semble pas avoir beaucoup changé depuis (Warshaw, 1989).

\section{Hésitations à interroger les femmes sur la violence conjugale}

Plusieurs personnes ne se sentent pas outillées pour aborder le sujet délicat de la violence conjugale avec leurs clientes. Les recherches de Craine et al. (1988), comme celles de Post et al. (1980) montrent que plusieurs femmes qui subissent de la violence de la part d'un conjoint et se retrouvent en psychiatrie ne sont pas identifiées comme des femmes violentées parce qu'on ne leur pose pas de questions à ce sujet. Les intervenantes et intervenants sociaux hésitent à cibler 
directement le problème de la violence conjugale. Ils se sentent plus à l'aise pour intervenir dans le cadre de thérapies familiales ou conjugales (Pâquet-Deehy, Rinfret-Raynor et Larouche, 1989).

Les personnes qui travaillent dans le domaine médical présentent des attitudes semblables (Raymond et Charbonneau, 1984). Comme le soulignait MacLeod en 1980, les médecins sont formés pour traiter les symptômes et non les expériences de vie de leurs patientes. Une recherche de Warshaw (1989) met en évidence que, même lorsqu'il est clair qu'une femme a été blessée par son partenaire, les professionnels de la santé qui la reçoivent en salle d'urgence lui posent rarement des questions directes sur cette agression. La grande majorité $(90 \%)$ des médecins de l'échantillon de Warshaw n'ont posé aucune question sur l'histoire psychosociale des femmes rencontrées. Ils $\mathrm{n}^{\prime}$ ont donc rien su de la violence physique subie par ces femmes ni de leurs conditions de vie et ils n'ont pas vérifié si elles étaient en sécurité. Dans $78 \%$ des cas, les médecins n’ont pas tenté de définir la relation qu'il y avait entre la victime et l'agresseur. Warshaw (1989) conclut qu'en ne posant pas ce genre de questions, la ou le médecin ne rate pas seulement l'occasion d'identifier la violence lorsque celle-ci n'est pas vraiment claire, mais il ne peut non plus s'engager dans aucune mesure préventive, ni protéger la femme dont la vie peut être en danger.

\section{Non-reconnaissance de la violence conjugale : impact sur l'intervention}

Sans reconnaître la présence de violence conjugale il est difficile de répondre de façon appropriée aux besoins des femmes violentées (Brekke, 1987 ; Kérouac et al., 1986; Kurz et Stark, 1988; Lewis, 1985 ; MacLeod, 1987 ; McCaughry et Sharp, 1991, Rose, Peabody, Stratigeas, 1991). Warren, Rose et Burgunder (1974) affirment même qu'un traitement inapproprié, prescrit dans l'ignorance de la violence subie, a nécessairement des conséquences négatives à court et à long terme.

L'ignorance du problème de la violence conjugale par les médecins ou psychiatres peut entraîner des erreurs de diagnostic et de traitement qui ont des conséquences désastreuses sur la santé mentale des femmes violentées (Rosewater, 1988). En effet, certaines femmes violentées font de longs séjours à l'hôpital psychiatrique et reçoivent des traitements pour différents malaises sans que la violence soit jamais identifiée (Craine et al., 1988). Des femmes violentées reçoivent des ordonnances de médicaments psychotropes (analgésiques, tranquillisants mineurs, etc.) pour les aider à mieux 
supporter le stress familial ou pour éliminer certains symptômes (perte d'appétit, insomnies, états dépressifs, etc.). Malheureusement, la violence, cause de ces symptômes, ne fait l'objet d'aucune intervention. Certains de ces médicaments peuvent rendre ces femmes encore plus vulnérables à la violence de leur partenaire (Stark et al., 1981), ou même conduire certaines d'entre elles au développement d'une dépendance ou d'une toxicomanie.

Les intervenantes et intervenants sociaux sont très souvent appelés à travailler auprès des couples ou des familles. Depuis quelques années, on commence à reconnaître que la violence conjugale est présente dans plusieurs couples qui demandent une thérapie familiale ou de couple. Selon Golden et Frank (1994), il est impératif de vérifier de façon sécuritaire la présence de violence, car omettre cette vérification peut mettre la femme en danger. En effet, une femme qui accepte, en thérapie, de parler ouvertement en présence de son partenaire peut être agressée à son retour à la maison (Jones et Schechter, 1992). Une femme violentée physiquement ou psychologiquement n'est pas libre de parler ou d'agir (Golden et Frank, 1994).

Golden et Frank (1994) considèrent que les thérapies familiales ou de couple sont inefficaces dans les situations de violence conjugale. D'une part, l'homme violent ne peut être un élément opérant de la cellule familiale tant qu'il maltraite sa femme ou ses enfants (Conseil du statut de la femme, 1978). D'autre part, la victime de violence $n^{\prime}$ est aucunement responsable des comportements de son partenaire et n'a pas le pouvoir de les changer. Selon Jones et Schechter (1982), I'effet des thérapies familiales ou de couple peut même se révéler négatif pour les femmes violentées, car ces thérapies risquent de rendre les femmes responsables de l'échec du couple ou de la famille et de les culpabiliser si elles tentent de se sortir de la situation de violence.

Les psychothérapies individuelles auprès des femmes violentées peuvent aussi comporter certains dangers si elles laissent entendre aux femmes que leurs difficultés relèvent d'un problème individuel ou intrapsychique (Lafrance, 1984) plutôt que d'identifier que l'homme violent est responsable de ses comportements. Ce type d'intervention peut renforcer le déni chez la femme victime de violence (Lewis, 1985), ou peut être interprété comme une forme d'acceptation implicite de cette violence (Brekke, 1987).

Rinfret-Raynor et al. (1992) ont réalisé une étude sur l'efficacité de I'intervention auprès des femmes violentées. Ces chercheuses ont identifié cinq conditions de base à l'efficacité de l'intervention : intervention centrée sur la femme plutôt que sur le couple ou la 
famille, accent placé sur la restauration de l'estime de soi, importance de l'aide concrète, travail sur les émotions liées à la violence et analyse féministe de la problématique. Cependant, pour réaliser une intervention efficace auprès des femmes violentées, il faut d'abord que la situation de violence conjugale soit reconnue.

\section{Dépistage systématique de la violence conjugale}

Selon Gendron (1987), peu d'outils permettent de repérer les femmes victimes de violence conjugale, avec pour conséquences que la violence $\mathrm{n}^{\prime}$ est pas identifiée et que les femmes violentées ne reçoivent pas les traitements et le soutien adéquats. Dans la plupart des établissements de santé et de services sociaux, il n'existe aucun système de dépistage permettant de distinguer les victimes de violence conjugale des autres clientèles (McCaughry et Sharp, 1991). Pourtant, depuis le début des années 80, des efforts de dépistage sont observés dans les différentes sphères d'intervention.

\section{Santé}

En 1982, une équipe de médecins du Conseil des soins de santé de I'Association médicale canadienne (Ghent, Da Sylva et Farren, 1982) a présenté un guide bilingue pour la détection de la violence au foyer et l'intervention en cette matière. Ce texte souligne que les intervenants et intervenantes du domaine de la santé sont souvent le premier et unique contact de la femme violentée avec des personnes étrangères à la famille, car plusieurs de ces femmes ne peuvent demander de l'aide que si cela se révèle nécessaire pour soigner des blessures. En plus de donner des pistes d'intervention, on encourage dans ce guide le dépistage de la violence conjugale en indiquant aux médecins les symptômes et les types de blessures que peuvent présenter les femmes violentées, et dont l'observation doit éveiller leurs soupçons.

En 1987, I'Ordre des infirmières et infirmiers du Québec a publié à l'intention de ses membres un document intitulé Écouter le langage des maux: la violence conjugale, intervention infirmière auprès des femmes. Plusieurs pages de ce document portent sur l'évaluation systématique de différents facteurs permettant aux infirmières et infirmiers de déceler les situations de violence conjugale. Une partie de ce document porte sur l'évaluation des risques d'homicide. II propose des pistes d'intervention auprès des femmes violentées. 


\section{Intervention sociale}

En janvier 1982, Ginette Larouche a préparé un Protocole d'intervention en service social auprès de la clientèle de femmes violentées qui traite de la situation de crise, de l'intervention à moyen terme et du dépistage. Ce texte affirme que "toute intervention sociale devrait comporter un dépistage de la violence conjugale» et suggère la vérification de plusieurs éléments, la présence d'un de ces éléments pouvant révéler un climat de violence. Ce premier protocole donne lieu, en 1985, à la publication par la Corporation des travailleurs sociaux du Québec d'un Guide d'intervention auprès des femmes violentées (Larouche, 1985). Une section complète de ce guide porte sur le dépistage. On y conseille de vérifier la présence de violence conjugale de façon systématique dans tous les dossiers nécessitant une intervention psychosociale. De plus, on propose d'évaluer le potentiel de violence par la vérification de certains facteurs: des indices individuels, des indices associés au couple et à la famille, ainsi que divers types de stress souvent associés aux comportements violents.

En 1985, une travailleuse sociale ontarienne (Sinclair, 1985) proposait un guide de formation, Pour comprendre le problème des femmes battues. En plus d'y définir et expliquer différentes formes de violence envers les femmes, elle décrit les objectifs de l'intervention et défait certains mythes sur la violence conjugale. Elle encourage les intervenants et les intervenantes à interroger les femmes sur la présence de violence et leur présente une liste d'indices pouvant les aider à dépister la violence conjugale.

En 1990, le Regroupement des CLSC du Montréal métropolitain a élaboré un programme de formation sur la violence conjugale pour toutes les personnes engagées dans tous les programmes de CLSC (auxiliaires familiales, infirmières, intervenantes et intervenants sociaux, médecins, réceptionnistes, etc.). Cette formation comprend un Guide de dépistage de la violence conjugale, un Guide d'animation sur le dépistage de la violence conjugale ainsi que deux vidéocassettes. Ce programme vise à faire du dépistage une priorité dans toute intervention infirmière, médicale ou psychosociale. En plus d'informer sur l'ampleur, les causes et les conséquences de la violence conjugale, ce guide de dépistage propose des principes de base d'intervention et apporte des précisions sur le rôle des différentes professions dans le dépistage de la violence conjugale. II n'offre cependant pas de moyens spécifiques et concrets pour dépister la violence.

Ces exemples, ainsi que plusieurs autres qui n'ont malheureusement pas pu être présentés ici, témoignent des efforts réels fournis 
dans différents milieux d'intervention pour encourager le dépistage de la violence conjugale. Pourtant, la violence conjugale demeure bien souvent cachée et trop de femmes violentées ne sont pas identifiées (Cantin et al., 1994 ; Rodgers, 1994). En fait, si le dépistage n'est pas réalisé de façon systématique et s'il ne s'appuie pas sur des instruments, il semble peu efficace (McCaughry et Sharp, 1991; Rodenburg et Fantuzzo, 1993). Il importe donc d'élaborer des outils de dépistage concrets qui permettent l'identification rapide des femmes victimes de violence conjugale afin de mieux les aider.

\section{DÉVELOPPEMENT DU PROTOCOLE DE DÉPISTAGE}

C'est dans ce contexte que le CLSC Saint-Hubert décide d'élaborer un outil stratégique pour identifier les femmes victimes de violence conjugale. Cet outil doit être accessible à l'ensemble des professionnels et s'intégrer facilement à leur travail quotidien. Le travail d'élaboration et d'évaluation de l'outil de dépistage est confié à une équipe de chercheuses du CRI-VIFF (Centre de recherche interdisciplinaire sur la violence familiale et la violence faite aux femmes) qui possèdent une expertise en matière de violence conjugale ainsi que dans l'évaluation des interventions.

Plusieurs étapes se sont succédé dans le développement du protocole de dépistage systématique de la violence conjugale: 1) des rencontres entre les chercheuses du CRI-VIFF et un comité multidisciplinaire du CLSC, 2) la réalisation d'une recension des écrits ${ }^{2}$, 3) l'étude de certains instruments de dépistage existants, et 4) la mise au point du protocole de dépistage.

\section{Première étape :}

\section{rencontres avec un comité multidisciplinaire du CLSC}

L'équipe de recherche a travaillé en collaboration étroite avec une équipe multidisciplinaire du CLSC formée de deux travailleuses sociales, d'une infirmière, $d^{\prime}$ une sexologue, d'une médecin et de la directrice des services à la clientèle. Cette collaboration a permis de définir certains besoins liés à l'intervention: I'outil mis en œuvre doit faire appel au jugement professionnel, sans obliger la cliente à

2. Cette recherche bibliographique fut réalisée grâce à l'obtention d'un fonds de démarrage du Centre de recherche interdisciplinaire sur la violence familiale et la violence faite aux femmes (CRI-VIFF). 
participer au processus de dépistage ; il doit être accessible à toutes les personnes intervenant auprès des femmes, peu importe leur formation et peu importe le contexte d'intervention (clinique avec rendez-vous, sans rendez-vous, services médicaux, infirmiers ou psychosociaux); il ne doit nuire d'aucune façon à la relation avec la cliente.

\section{Deuxième étape : recension des écrits sur le dépistage de la violence conjugale}

Les écrits sur la violence conjugale ont fourni plusieurs éléments à la compréhension du dépistage de la violence conjugale. Plusieurs écrits sur l'importance du dépistage, les difficultés liées au dépistage et les conséquences du manque de dépistage sur la santé physique et mentale des femmes et des enfants ont renforcé la démarche amorcée par le CLSC. La rareté des outils ainsi que I'absence d'évaluation de l'efficacité des instruments existants ont confirmé la nécessité de mener à terme un projet de partenariat entre le milieu de l'intervention et celui de la recherche afin d'élaborer et d'évaluer des outils.

\section{Troisième étape : étude des instruments de dépistage existants}

La recension des écrits a permis d'identifier les différents moyens de dépistage de la violence conjugale déjà existants. Cette recherche, complétée par des contacts avec des personnes-ressources de différents organismes, a permis de répertorier une quinzaine d'outils de dépistage (Brekke, 1987; Cantin, 1986; Clément et Ouellet, 1992; Gendron, 1991; Ghent, Da Sylva et Farren, 1982; Hudson et McIntosh, 1981; Larouche, 1985; Lewis, 1985; Marshall, 1992; McCaughry et Cameron, 1994 ; Ordre des infirmières et infirmiers du Québec, 1987 ; Regroupement des CLSC du Montréal métropolitain, 1990 ; Rinfret-Raynor et al., 1991 ; Rosewater, 1985 ; Sinclair, 1985; Straus, Gelles et Steinmetz, 1980).

Parmi les outils de dépistage répertoriés, douze instruments (six en français et six en anglais) ont été remis pour étude à l'équipe multidisciplinaire. On compte parmi ces instruments huit questionnaires, deux protocoles d'identification des facteurs de risque liés à la violence, un protocole d'entrevue et un instrument combinant observation et entrevue. Mis à part les questionnaires qui ont tous fait l'objet de certaines études de validation présentant la structure factorielle ou la cohérence interne de ces instruments, peu d'études 
évaluent leur efficacité à dépister la violence conjugale (validité prédictive), et aucun des documents répertoriés n'offre une évaluation de leurs effets sur les femmes dépistées ou sur l'intervention auprès de ces femmes après le dépistage. On constate donc une absence de données sur les effets de l'utilisation systématique d'un protocole de dépistage de la violence conjugale.

\section{Quatrième étape: mise au point d'un premier protocole de dépistage}

L'équipe du CLSC et l'équipe de recherche optent finalement pour un protocole de dépistage formé de deux instruments. Celui-ci comporte une grille d'observation qui est suivie, lorsque cette grille laisse présager la présence de violence conjugale, d'un questionnaire rempli avec la cliente.

\section{Premier instrument du protocole:}

\section{Grille d'évaluation de la violence conjugale (GÉV)}

Un apprentissage commun à plusieurs disciplines et l'observation de différents signes peuvent révéler la présence de différents problèmes. Une grille d'observation permet de systématiser ce mode de dépistage utilisé de façon informelle en offrant un moyen concret pour noter rapidement ces observations et les garder ainsi disponibles pour appuyer leur intervention.

La Grille d'évaluation de la violence (GÉV) est conçue de façon à offrir la possibilité de consigner les signes de violence conjugale observés chez les clientes. L'idée de cette grille s'inspire du travail de Clément et Ouellet (1992) qui ont construit deux grilles pour évaluer le degré de risque de violence envers les personnes âgées.

La GÉV a donc pour objectif d'évaluer le degré de risque de violence conjugale. Son élaboration s'appuie sur l'hypothèse suivante: la violence ayant de nombreux effets sur les femmes qui en sont victimes, certains indices, décelables dans le discours, les comportements ou l'état de santé des femmes violentées, devraient permettre de présumer une situation de violence conjugale. La GÉV a été élaborée à partir d'un examen attentif des travaux sur les conséquences de la violence conjugale sur les femmes violentées et les facteurs de risques sociaux et médicaux associés à la violence conjugale. Plus de 150 éléments, fréquemment présents en situation de violence conjugale, ont été compilés pour former une grille comportant 76 de ces signes regroupés en quatre parties. 
- PARTIE I

INDICATEURS POUVANT RÉVÉLER LA PRÉSENCE DE VIOLENCE CONJUGALE

La première partie de la GÉV comporte 19 indicateurs pouvant révéler la présence de violence conjugale: certaines blessures suspectes (par exemple, une blessure aux organes génitaux), des explications incongrues (par exemple, une description de l'accident qui ne coïncide pas avec le type de blessure) et certains comportements de la femme ou de son partenaire (par exemple, la femme regarde constamment son partenaire avant de répondre). Les indicateurs de cette partie sont suffisamment importants pour que la présence d'un seul d'entre eux appelle une vérification de la présence de violence.

- PARTIE II

SIGNES PHYSIQUES POUVANT RÉVÉLER LA PRÉSENCE DE VIOLENCE CONJUGALE

La seconde partie comprend 16 signes physiques (par exemple, fracture ou dislocation de la mâchoire) et un comportement (visites fréquentes au CLSC). Ces signes ne sont pas propres à la violence conjugale, mais y sont souvent rattachés.

- PARTIE III

DIFFICULTÉS DANS LA RELATION DE COUPLE, SITUATIONS DANGEREUSES ET CONDITIONS SOCIALES FRÉQUEMMENT ASSOCIÉES À LA VIOLENCE CONJUGALE

La troisième partie comporte 18 éléments: certaines difficultés dans la relation de couple (par exemple, I'homme dicte la conduite de sa partenaire), certains comportements du partenaire (par exemple, il est violent envers les enfants) et certaines situations et conditions sociales fréquemment associées à la violence conjugale (par exemple, isolement social). Ces indicateurs ne sont pas propres à la violence conjugale, mais y sont souvent rattachés.

- PARTIE IV

SIGNES D'UN ÉTAT ÉMOTIONNEL POUVANT RÉVÉLER LA PRÉSENCE DE VIOLENCE CONJUGALE

La quatrième partie présente 22 signes d'un état émotionnel de la femme pouvant découler de la violence conjugale (par exemple, elle semble triste, déprimée ou fragile émotivement). Ces signes peuvent être présents chez certaines femmes qui ne subissent pas la violence de leur partenaire, mais comme ils sont souvent rattachés à la violence conjugale, ils peuvent éveiller des soupçons. 


\section{UTILISATION DE LA GÉV}

La GÉV est déposée par les personnes des deux groupes dans le dossier des femmes qui utilisent les services du CLSC, et peut être remplie après chaque contact avec elles. Cette grille est utilisée dès la première rencontre, immédiatement après l'entrevue. Elle ne nécessite pas la participation de la cliente. En plus d'identifier dans la grille les éléments observés, la personne qui I'utilise peut aussi commenter ses observations.

Si un élément de la partie I ou plusieurs éléments des parties II, III ou IV sont identifiés (le nombre est laissé au jugement de I'intervenante), il est possible que la femme subisse une certaine forme de violence de la part de son partenaire. La présence de violence pourra être vérifiée en posant une question claire à la cliente en l'absence du partenaire. Cette question est laissée à la discrétion de l'intervenante ou de l'intervenant, qui peut par exemple lui demander si son partenaire lui a déjà fait mal physiquement. Si la femme ne répond pas ou répond négativement à cette question, le second instrument est utilisé.

\section{Deuxième instrument du protocole:} questionnaire rempli avec la cliente

Lorsque la grille d'observation révèle la présence d'un nombre suffisant de facteurs de risque, le dépistage se poursuit par l'utilisation $d^{\prime}$ un questionnaire rempli oralement avec la cliente.

Pour cette deuxième partie du protocole, I'équipe CLSC / CRIVIFF a retenu deux instruments : I'Instrument d'identification des femmes violentées en milieu conjugal (IIFVMC) élaboré par Gendron (1991) et le Wife Abuse Inventory (WAI) de Lewis (1985), dans sa version française (McCaughry et Sharp, 1991). Ces deux instruments offrent I'avantage d'être assez courts, d'aborder différents types de problèmes dans le couple et de poser des questions qui, sans porter directement sur les comportements violents du conjoint, ont démontré leur potentiel pour susciter un questionnement chez la femme qui y répond. Ils permettent tous les deux d'obtenir un score total qui reflète la probabilité que la répondante soit violentée par son partenaire. De plus, une partie au moins de la validation de ces deux instruments a été réalisée auprès de la clientèle des CLSC.

- INSTRUMENT D'IDENTIFICATION DES FEMMES VIOLENTÉES EN MILIEU CONJUGAL

L'Instrument d'identification des femmes violentées en milieu conjugal (IIFVMC) élaboré par Gendron (1991) a été bâti à partir 
de travaux en sciences infirmières, particulièrement les écrits de Campbell (1989) et de Orem (1985). L'échelle comporte 30 items regroupés autour de quatre facteurs de croissance personnelle: estime de soi, autonomie, sociabilité et sécurité. Chaque question s'accompagne d'une échelle de type Likert à cinq niveaux ( 1 : entièrement $d^{\prime}$ accord à 4 : entièrement en désaccord et $5:$ ne s'applique pas).

La validation de I'instrument (Gendron, 1991) a permis de constater que I'IIFVMC présente une bonne capacité pour discriminer si les femmes sont violentées ou non et l'analyse $d^{\prime}$ items montre que l'échelle présente un coefficient alpha de 0,975 . L'auteure souligne qu'en plus de permettre l'identification des femmes violentées, le questionnaire sert de soutien à la discussion entre la répondante et l'intervenante et permet aux femmes de prendre conscience du climat de violence dans lequel elles vivent.

- Version fRANÇAISE dU WIFE AbUSE INVENTORY

La version française du Wife Abuse Inventory (WAI) comporte 25 items qui tentent de mesurer trois facteurs de risque associés à la violence conjugale: 1) l'image de soi du partenaire, 2) I'isolement social de la famille et 3) le mode habituel de résolution de conflits utilisé au sein de la famille. Chaque question s'accompagne d'une échelle de type Likert à quatre échelons $(1$ : rarement ou jamais à 4 : toujours). Cette échelle permet de calculer le WAISCORE et ainsi d'établir le niveau de risque de violence.

Comme le WAI permet d'identifier certaines zones de vulnérabilité, il peut constituer un bon indicateur pour l'intervention (McCaughry et Sharp, 1991). De plus, il permet de déterminer le risque de violence, indépendamment du degré de conscience que la victime de violence peut avoir de sa condition. McCaughry et Cameron (1994) considèrent qu'un outil de dépistage comme le WAI peut permettre d'intervenir sur les facteurs prédictifs de risque avant l'apparition des comportements de violence, et peut servir d'instrument de sensibilisation auprès des femmes qui ne considèrent pas que certains comportements de leur conjoint constituent de la violence.

Un prétest (McCaughry et Sharp, 1991) a permis d'éprouver la validité nominale et de contenu du WAI ainsi que sa capacité discriminante et prédictive. L'application de l'instrument à 562 femmes a confirmé la capacité discriminante du WAI. 


\section{Deux protocoles}

Deux protocoles de dépistage de la violence conjugale sont donc constitués. Comme le présente la figure 1 , chacun de ces protocoles comprend la Grille d'évaluation de la violence conjugale (GÉV). Si plusieurs indices notés sur cette grille laissent présager la présence de violence conjugale, le questionnaire est utilisé. Le groupe 1 utilise alors le IIFVMC et le groupe 2 le WAI.

\section{Figure 1}

Vue d'ensemble des deux protocoles de dépistage de la violence conjugale

\section{Groupe 1}

Utilisation

du premier protocole

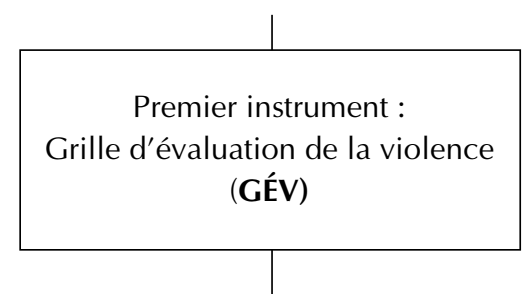

Si plusieurs indices

\begin{tabular}{|c|}
\hline Deuxième instrument : \\
Questionnaire \\
Instrument d'identification \\
des femmes violentées \\
en milieu conjugal \\
\hline
\end{tabular}

Groupe 2

Utilisation

du second protocole

Premier instrument :

Grille d'évaluation de la violence

(GÉV)

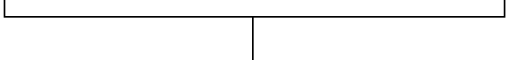

Si plusieurs indices

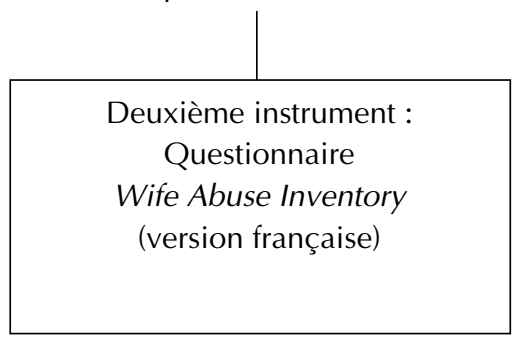

\section{EXPÉRIMENTATION DES PROTOCOLES}

Pour expérimenter les protocoles, deux équipes multidisciplinaires sont formées au sein des services courants du CLSC. La première équipe, composée de trois travailleuses sociales, deux infirmières et deux médecins, utilise le premier protocole (GÉV et IIFVMC). La seconde équipe, composée de deux infirmières, deux médecins, une sexologue et une travailleuse sociale, utilise le second protocole (GÉV et WAI). 


\section{Formation}

Chaque groupe reçoit une formation préparatoire à l'utilisation des protocoles. Cette formation porte sur la problématique de la violence conjugale, sur les difficultés d'identification des femmes victimes de violence conjugale ainsi que sur la façon d'utiliser les instruments de dépistage proposés.

Pendant toute la durée de l'expérimentation, les deux équipes sont supervisées par une coordonnatrice du CLSC et une chercheuse du CRI-VIFF.

\section{Utilisation des protocoles et suivi}

La période d'expérimentation s'étale sur trois mois, soit du 21 mars 1994 au 20 juin 1994. Pendant cette période, le protocole de dépistage est utilisé auprès de toutes les usagères rencontrées sur rendez-vous aux services courants du CLSC pour consultation psychosociale, infirmière ou médicale.

Pour permettre une familiarisation progressive avec les instruments du protocole, pendant les deux premières semaines $d^{\prime}$ utilisation seule la GÉV est remplie. Après cette période, le protocole complet est utilisé auprès de toutes les femmes rencontrées sur rendez-vous. À la fin de l'expérimentation, tous les instruments sont retirés des archives et remis aux chercheuses.

Après un mois d'utilisation, des rencontres de suivi avec les deux groupes ont eu lieu. Les aspects techniques de I'utilisation des protocoles ont été vérifiés et les difficultés d'utilisation ont été relevées et corrigées. Ces rencontres ont aussi permis de rappeler certaines consignes.

\section{Résultats de l'expérimentation ${ }^{3}$}

Il importe de rappeler que la présente étude constitue en fait une étape préalable à l'utilisation d'un protocole de dépistage systématique de la violence conjugale dans tous les services du CLSC SaintHubert. Afin de mettre directement en lumière les constats généraux qui se sont imposés, les résultats de cette préexpérimentation sont présentés, analysés et interprétés simultanément.

3. L'analyse des données de la préexpérimentation a été réalisée grâce à une subvention du Conseil québécois de la recherche sociale. 
Quatre moyens permettent d'évaluer les résultats de la préexpérimentation.

1. Des entrevues de groupe sont réalisées avec les membres des deux équipes multidisciplinaires qui ont utilisé les protocoles de dépistage. Une seule intervenante est absente à ces rencontres pour cause de maladie.

2. Un questionnaire est remis aux personnes des deux équipes. Ce questionnaire a été bâti spécifiquement pour connaître leurs impressions sur le protocole de dépistage. En tout, six questionnaires ont été compilés. Parmi les personnes qui ont répondu, deux avaient utilisé le IIFVMC (première équipe), trois avaient utilisé le WAI (deuxième équipe) et une personne a omis d'indiquer à quel groupe elle appartenait.

3. Après les entrevues de groupe et le dépouillement des questionnaires, il a semblé nécessaire de réaliser des entrevues individuelles pour connaître davantage les impressions sur les instruments. La majorités des personnes des deux équipes, sauf trois qui sont en congé, sont interviewées. De plus, des entrevues sont réalisées avec deux cadres qui participent à la supervision des équipes.

4. Les 182 protocoles utilisés pendant les trois mois d'utilisation sont analysés.

Les informations obtenues par ces différents moyens sont regroupées en trois thèmes: le protocole de dépistage en général ; la grille d'observation; les questionnaires (IIFVMC et WAI).

\section{Le protocole de dépistage en général}

La majorité des intervenants et intervenantes constatent que I'utilisation du protocole favorise le dépistage de la violence conjugale. De plus, certaines personnes considèrent que les outils de dépistage les soutiennent dans leur intervention et leur donnent des assises plus solides pour confirmer les appréhensions de violence conjugale.

Lors des rencontres de groupe, les protocoles utilisés par les deux équipes sont jugés faciles d'application. Cependant, les données recueillies par les questionnaires et les entrevues individuelles permettent de réaliser que dans la pratique des difficultés apparaissent à cause du peu de temps disponible pour compléter les instruments. Pendant les dernières semaines de la période d'expérimentation, 
certaines personnes ont décidé d'arrêter de remplir les grilles et de répondre aux questionnaires, parce que le temps leur manquait pour le faire. Il importe donc de mieux adapter les instruments à la réalité de l'intervention au CLSC.

Les membres des deux groupes considèrent que I'application des protocoles de dépistage entraîne un certain alourdissement de leur tâche. Cet alourdissement serait attribuable aux nombreux aspects techniques reliés à leur utilisation: identification des dossiers, accès aux instruments, etc. Plusieurs de ces problèmes découlent du nombre restreint de personnes engagées dans l'utilisation expérimentale des protocoles. Ces côtés techniques astreignants disparaîtront lorsque l'utilisation des instruments sera généralisée à tout le CLSC.

La majorité des membres des deux équipes ont trouvé l'expérience positive et stimulante, bien que trop courte pour bien intégrer les instruments. Ces personnes déplorent cependant un manque de formation: trop peu de temps fut consacré à l'apprentissage et à la maîtrise des instruments.

\section{La grille d'observation}

La grille d'observation a été généralement appréciée. Elle permet de garder à l'esprit l'objectif de dépistage. Certaines personnes mentionnent qu'une consigne plus précise quant au nombre de signes nécessaires pour passer au second instrument leur aurait évité d'hésiter avant d'utiliser le questionnaire.

Certaines personnes trouvent la GÉV longue et lourde. Cependant, la majorité se dit généralement plus portée à l'utiliser que le questionnaire. La partie IV, qui présente des signes d'un état émotionnel pouvant révéler la présence de violence conjugale, est particulièrement appréciée par la majorité des intervenants et intervenantes des deux groupes. Plusieurs rapportent que l'observation de ces signes leur a permis de dépister des victimes de violence conjugale.

Ces commentaires sont confirmés par la compilation des protocoles. Pendant les trois mois d'expérimentation, 182 grilles d'observation (GÉV) ont été remplies (comparativement à 10 questionnaires). Elles ont été utilisées par les membres de toutes les formations (médecine, sciences infirmières et intervention sociale).

Le tableau 1 montre que, parmi les 76 items de la GÉV, 47 ont été observés au moins une fois chez les femmes rencontrées pendant cette période, alors que 29 items n'ont jamais été observés. 
Tableau 1

Fréquence d'utilisation des éléments de la GÉV

\begin{tabular}{lcccc}
\hline & $\begin{array}{c}\text { Nbre d'items } \\
\text { disponibles }\end{array}$ & $\begin{array}{c}\text { Nbre d'items } \\
\text { utilisés }\end{array}$ & $\begin{array}{c}\text { Nbre d'items } \\
\text { utilisés plus } \\
\text { de 10 fois }\end{array}$ & $\begin{array}{c}\text { Nbre total } \\
\text { d'utilisation } \\
\text { des items }\end{array}$ \\
\hline $\begin{array}{l}\text { PARTIE I } \\
\text { Indicateurs pouvant } \\
\text { révéler la présence } \\
\text { de violence conjugale }\end{array}$ & $\mathbf{1 9}$ & $\mathbf{5}$ & $\mathbf{0}$ & $\mathbf{6}$ \\
$\begin{array}{l}\text { PARTIE II } \\
\text { Signes physiques pouvant } \\
\text { révéler la présence } \\
\text { de violence conjugale }\end{array}$ & $\mathbf{1 7}$ & $\mathbf{3}$ & $\mathbf{0}$ & $\mathbf{6}$ \\
$\begin{array}{l}\text { PARTIE III } \\
\text { Difficultés dans la relation } \\
\text { de couple, situations } \\
\text { dangereuses et conditions } \\
\text { SOciales fréquemment } \\
\text { associées à la violence } \\
\text { conjugale }\end{array}$ & $\mathbf{1 8}$ & $\mathbf{1 8}$ & $\mathbf{3}$ & $\mathbf{1 2 8}$ \\
$\begin{array}{l}\text { PARTIE IV } \\
\text { Signes d'un état émotionnel } \\
\text { pouvant révéler la présence } \\
\text { de violence conjugale }\end{array}$ & $\mathbf{2 2}$ & & & \\
\hline \begin{tabular}{l} 
Total \\
\hline
\end{tabular} & $\mathbf{7 6}$ & $\mathbf{4 7}$ & $\mathbf{1 3}$ & $\mathbf{3 9 9}$ \\
\hline
\end{tabular}

On peut constater que les éléments des deux premières parties de la GÉV ont été peu observés: seulement cinq indicateurs de la partie I et trois signes physiques de la partie II ont été cochés. Par contre, tous les indicateurs de la partie III et tous les signes émotionnels de la partie IV sauf un ont été utilisés au moins une fois. Treize signes ont été observés plus de dix fois, l'élément utilisé le plus fréquemment (la femme semble manquer d'estime d'elle-même) ayant été observé chez 28 femmes.

L'âge moyen des 182 femmes de l'échantillon est de 34 ans. La plus jeune a 15 ans et l'aînée 78 ans. Pendant l'expérimentation, la violence conjugale a été confirmée pour 24 femmes. Il est cependant difficile de déterminer combien de femmes le protocole a réellement permis de dépister. En effet, les entrevues individuelles ont révélé que certaines personnes ont utilisé la grille avec des femmes dont la situation de violence conjugale était déjà connue. En interrogeant sur le nombre de victimes de violence conjugale dépistées, on 
parvient à estimer que 11 femmes auraient réellement été dépistées grâce à la grille.

En répartissant le nombre de grilles en fonction du nombre de signes identifiés sur chacune d'elles, on constate que 75 grilles ont permis l'observation d'au moins un signe. Certaines femmes présentent cependant plusieurs signes: chez 45 femmes, quatre signes et plus ont été observés, alors que 8 femmes présentaient plus de dix signes.

Le tableau 2 permet de comparer le nombre total de signes observés chez les 24 femmes pour lesquelles la violence est confirmée, par rapport au nombre de signes observés chez celles pour qui la violence $n^{\prime}$ est pas dépistée $(n=158)$. La grille d'observation semble avoir été un bon soutien au dépistage, puisque la violence conjugale se confirme davantage chez les femmes qui présentent plusieurs signes. Les femmes violentées présentent en moyenne 7,75 signes, alors que les autres femmes présentent 1,35 signe seulement. Cette différence est statistiquement significative $\left(K h i^{2}=389,5\right)$.

TABLeAu 2

Fréquence d'observation des éléments de la GÉV chez les femmes pour qui la violence conjugale est confirmée ou non

\begin{tabular}{lc|cc|cc}
\hline & & \multicolumn{2}{|c|}{$\begin{array}{c}\text { Violence confirmée } \\
\text { (24 sujets) }\end{array}$} & $\begin{array}{c}\text { Violence non confirmée } \\
\text { (158 sujets) }\end{array}$ \\
\hline $\begin{array}{l}\text { Nombre total } \\
\text { de signes } \\
\text { observés }\end{array}$ & $\begin{array}{c}\text { Nombre de signes } \\
\text { observés }\end{array}$ & $\begin{array}{c}\text { Nombre moyen } \\
\text { de signes } \\
\text { par sujet }\end{array}$ & $\begin{array}{c}\text { Nombre de signes } \\
\text { observés }\end{array}$ & $\begin{array}{c}\text { Nombre moyen } \\
\text { de signes } \\
\text { par sujet }\end{array}$ \\
\hline PARTIE I & 6 & 5 & 0,21 & 1 & 0,01 \\
PARTIE II & 6 & 0 & 0,00 & 6 & 0,04 \\
PARTIE III & 128 & 82 & 3,42 & 46 & 0,29 \\
PARTIE IV & 259 & 99 & 4,13 & 160 & 1,01 \\
\hline Total & $\mathbf{3 9 9}$ & $\mathbf{1 8 6}$ & $\mathbf{7 , 7 5}$ & $\mathbf{2 1 3}$ & $\mathbf{1 , 3 5}$ \\
\hline
\end{tabular}

\section{Les questionnaires}

Les questionnaires ont été peu utilisés. Pendant les trois mois de cette expérimentation, seulement dix ont été remplis. Le IIFVMC a été utilisé quatre fois et le WAI six fois.

Plusieurs personnes estiment que les questionnaires sont difficiles à utiliser comme outils de dépistage, particulièrement dans une clinique $d^{\prime}$ urgence où les ressources et le temps sont restreints. Elles souhaitent obtenir des outils de dépistage plus sensibles et moins intrusifs que les questionnaires. 
Certaines personnes les jugent cependant bénéfiques comme outils d'intervention lorsqu'ils sont utilisés dans le but de confirmer aux clientes la présence de violence conjugale. Les entrevues individuelles ont d'ailleurs permis de réaliser que les questionnaires ont souvent été remplis dans un but d'intervention plutôt que de dépistage. En fait, deux questionnaires seulement ont réellement été utilisés dans un but de dépistage, pour vérifier une appréhension de violence conjugale. Ils ont été efficaces, puisque les cotes obtenues étaient au-dessus du seuil critique et indiquaient la présence de violence, et que, dans les deux cas, les femmes ont par la suite confirmé la présence de violence.

Même en les utilisant comme outils d'intervention, les scores obtenus par les questionnaires confirment toujours la violence conjugale lorsque celle-ci est présente. On a répondu à deux questionnaires sans que la grille d'observation soit préalablement remplie. Ces personnes ont donc utilisé directement le questionnaire pour cerner, avec la femme, la situation de violence, même si celle-ci était déjà clairement identifiée.

Cette préexpérimentation a constitué une étape très importante de l'élaboration du protocole de dépistage. À partir de ces résultats, le protocole a été transformé et son utilisation généralisée à I'ensemble du CLSC, dans le cadre d'une recherche évaluative ${ }^{4}$ reliée à I'implantation d'un nouveau protocole de dépistage. Cette recherche, d'une durée de trois ans, comporte un volet d'évaluation formative et un volet d'évaluation sommative. Elle vise à évaluer l'applicabilité du protocole de dépistage et à connaître son efficacité, de même que ses effets sur la clientèle, sur l'intervention et sur l'organisation.

\section{CONCLUSION}

L'utilisation expérimentale du protocole de dépistage a donné lieu à plusieurs constats. Certains concernent le protocole de dépistage, $\mathrm{d}^{\prime}$ autres la démarche de partenariat entre le milieu de recherche et le milieu d'intervention.

\section{Protocole de dépistage}

À la suite de l'analyse des données recueillies, le protocole a pu être transformé et adapté pour en faire un outil plus souple et plus facile d'application. Ce protocole transformé comporte trois parties.

4. Cette recherche est subventionnée par le CQRS et le CRSH. 
Comme la grille d'observation (GÉV) a été généralement appréciée et fréquemment utilisée pendant la préexpérimentation et comme elle a facilité le dépistage, elle constitue encore la première partie du protocole. Des modifications importantes y ont cependant été apportées. Beaucoup d'items furent éliminés, seuls les indicateurs discriminants étant conservés. Le nombre de signes nécessaires pour passer à l'étape suivante a été précisé: on considère que l'observation de quatre ou cinq signes doit suffire pour éveiller les soupçons et pour poursuivre le dépistage en utilisant la deuxième partie du protocole.

Lorsque le nombre de signes de la GÉV laisse présager la présence de violence conjugale sans que celle-ci soit confirmée, un processus d'entrevue de dépistage permet ensuite de discuter de la gestion des conflits dans le couple. C'est la deuxième partie du protocole.

Si la violence n'est pas confirmée et si des doutes subsistent après cette entrevue, le dépistage de la violence conjugale est ensuite complété par un questionnaire qui permet de poser aux femmes des questions précises sur les comportements de leur partenaire. Ce questionnaire, troisième partie du protocole, s'inspire du Conflict Tactic Scales de Straus, Gelles et Steinmetz (1980).

Les modifications du protocole original visaient à s'assurer que le nouveau protocole utilisé au CLSC Saint-Hubert corresponde réellement aux besoins des personnes qui I'utiliseront quotidiennement. Avant de le mettre à la disposition de tous les intervenants et intervenantes des services courants, le nouveau protocole a été présenté aux membres des deux équipes de la préexpérimentation qui l'ont évalué très positivement. Plusieurs ont alors exprimé leur satisfaction en soulignant que ce nouveau protocole tenait compte des besoins exprimés à la suite de la préexpérimentation.

\section{Démarche en partenariat}

La collaboration étroite entre l'équipe des chercheuses et les intervenants du CLSC St-Hubert a permis de mener une réflexion sur le partenariat de recherche tel qu'il a été expérimenté dans cette préexpérimentation. La recherche en partenariat exige des personnes participantes une capacité d'écoute et d'adaptation fort différente de celle requise dans les projets de recherche plus traditionnels. Travailler en partenariat comporte beaucoup d'ajustements, des apprentissages et des défis pour tous les partenaires.

Pour les chercheuses, la réalisation d'activités de recherche en partenariat avec un milieu d'intervention appelle une ouverture aux nombreux questionnements sur la définition même de l'objet de 
recherche et sur les pratiques de cette recherche, y compris les aspects méthodologiques et déontologiques. Ces questionnements les obligent à revoir différents aspects de la recherche et à parfois les modifier.

Le travail de recherche en partenariat modifie également certaines habitudes quotidiennes des membres de l'équipe de recherche. Par exemple, il importe pour les gens du milieu d'intervention que la recherche soit visible sur le terrain, ce qui change de la recherche traditionnelle qui se réalise généralement dans les laboratoires de I'université.

Un très grand défi posé à l'équipe de recherche dans un tel partenariat est celui de devoir harmoniser les exigences de rigueur méthodologique de la recherche avec celles de la pratique du milieu d'intervention. Pour y parvenir, les membres de l'équipe doivent obligatoirement connaître les pratiques et les modes d'intervention du milieu terrain et $s^{\prime} y$ adapter. Toute recherche qui ne saurait respecter les pratiques et qui aurait pour effet de les modifier afin de les évaluer se situerait à l'encontre des objectifs de partenariat.

Si la recherche en partenariat pose un certain nombre de défis à la recherche, elle en pose également plusieurs aux milieux $d^{\prime}$ intervention. Il a été mentionné que les praticiennes et les praticiens remettent en question les méthodes de recherche. De la même façon, l'arrivée d'une équipe de recherche au sein d'un organisme d'intervention bouleverse certaines habitudes. Inévitablement, les chercheuses s'interrogent sur les pratiques, ne serait-ce que pour mieux les comprendre. Même si la présence des chercheuses dans l'établissement favorise leur intégration au sein du personnel et stimule la collaboration, elle n'est pas sans interpeller les personnes du milieu de pratique dans leur travail quotidien.

Dans cette expérience avec le CLSC Saint-Hubert, la collaboration du conseil d'administration et de la direction du CLSC a été essentielle à la réussite de la recherche. Cette collaboration s'est manifestée par un engagement à donner la priorité à toutes activités liées à la recherche, un soutien constant à l'équipe de recherche et une capacité de demeurer constamment informé du déroulement des activités de recherche. De plus, I'implication de ces instances a permis de répondre aux préoccupations des intervenants et des intervenantes au regard de la recherche et par le fait même d'en faciliter la réalisation. C'est dans cette perspective que le CLSC a obtenu un avis juridique pour répondre à des questions déontologiques concernant le fait de dépister systématiquement la violence conjugale chez toutes les femmes qui s'adressent aux services courants. Cet avis a 
permis de s'assurer que les droits des clientes du CLSC sont toujours respectés.

Par ailleurs, cette expérience de partenariat entre l'équipe de chercheuses et le CLSC a démontré l'importance de définir très clairement plusieurs aspects de la pratique tenus pour acquis dans toutes interventions. Ainsi, même les notions de dépistage et de violence conjugale peuvent prendre des sens très différents et ont dû être clarifiées dans la démarche de recherche.

Mentionnons, enfin, que dans certaines situations les pratiques administratives doivent également être mises au service de la recherche. Dans cette perspective, pour que l'efficacité du dépistage puisse être évaluée le CLSC a dû standardiser certaines méthodes de travail, revoir l'organisation de ses services à la clientèle ainsi que le système de collecte de données statistiques.

Tous ces efforts et un engagement soutenu des chercheuses et des intervenantes et intervenants leur ont permis de surmonter ensemble les obstacles inévitables de ce genre de recherche. Ils ont ainsi pu atteindre finalement l'objectif d'élaborer un protocole de dépistage systématique de la violence conjugale qui corresponde réellement aux besoins des personnes qui l'utilisent. 


\section{Références bibliographiques}

ADSHEAD, G. (1994). "Damage: Trauma and Violence in a Sample of Women Referred to a Forensic Service», Behavioral Sciences and the Law, vol. $12: 235-249$.

Aguilar, R.J. et N.N. Nightingale (1994). "The Impact of Specific Battering Experiences on the Self-Esteem of Abused Women », Journal of Family Violence, vol. 9, $\mathrm{n}^{\circ} 1: 35-45$.

AMERICAN PSYCHIATRIC ASSOCIATION (1994). Diagnostic and Statistical Manual of Mental Disorders (4 éd.). Washington, D.C.

BLOOM, M. (1987). "Toward a Technology in Primary Prevention: Educational Strategies and Tactics», Journal of Primary Prevention, vol. 8, $\mathrm{n}^{\circ} 1-2: 25-46$.

BREKKE, J.S. (1987). "Detecting wife and child abuse in clinical settings", Social Casework, vol. 68, $\mathrm{n}^{\circ} 6: 332-338$.

CAMPBELL, J.C. (1989). "A test of two explanatory models of women's response to battering », Nursing Research, vol. 38, n $1: 18-24$.

CANTIN, S., M. RINFRET-RAYNOR et L. FORTIN (1994). Utilisation des ressources par les victimes de violence conjugale. Collection Études et analyses, $\mathrm{n}^{\circ} 1$. Montréal: CRI-VIFF.

CARRIER, M. et N. MICHAUD (1982). La violence faite aux femmes en milieu conjugal: le produit d'une société sexiste, une étude québécoise. Ottawa: Secrétariat d'État, Programme pour la situation de la femme.

ChÉNARD, L., H. CADRIN et J. LOISELLE (1990). État de santé des femmes et des enfants victimes de violence conjugale. Rapport de recherche. Département de santé communautaire, Centre hospitalier régional de Rimouski.

CLÉment, M. et H. Ouellet (1992). «La violence n'a pas d'âge: prévenir et dépister les abus chez les personnes âgées », Intervention, 92, 63-75.

COMité sur la SAnté MENTALE du QuÉBEC (1993). Le défi de l'égalité : la santé mentale des hommes et des femmes. Boucherville: Gaëtan Morin Éditeur.

CONFÉREnCE NATiONALE SUR LA ViOlenCE FAMILIALE (1989). Main dans la main : Procès verbaux. Ottawa.

COnSEIl Du STAtut de LA fEMme (1978). Pour commencer, parlons-en... Québec.

COnseil du Statut de la femme (1993). Pour que cesse l'inacceptable: Avis sur la violence faite aux femmes. Québec.

CRAINE, L.S., C.E. HeNSON, J.A. COLLIVER et D.G. Maclean (1988). «Prevalence of a history of sexual abuse among female psychiatric patients in a state hospital system », Hospital and Community Psychiatric, 39, 300-304.

DUTTON, D.G. et S. PAINTER (1993). "The battered woman syndrome: effects of severity and intermittency of abuse », American Journal of Orthopsychiatry, vol. 63, $\mathrm{n}^{\circ} 4: 614-622$.

Dutton, M.A. et L.A. GoOdman (1994). "Posttraumatic Stress Disorder Among Battered Women : Analysis of Legal Implications», Behavioral Sciences and the Law, 12, 215-234. 
Follingstad, D.R., A.F. Brennan, E.S. Hause, D.S. Polek et L.L. Rutledge (1991). "Factors Moderating Physical and Psychological Symptoms of Battered Women », Journal of Family Violence, vol. 6, $\mathrm{n}^{\circ} 1$ : 81-95.

FRANKEL-HOWARD, D. (1989). La violence familiale : Examens des écrits théoriques et cliniques. Ottawa : Ministère des Approvisionnements et Services.

GAUTHIER, A. (1991). Intervention auprès des conjoints violents : Contre Toutes Agressions Conjugales (C-TA-C). Ottawa : Solliciteur général du Canada.

Gendron, C. (1987). Violence conjugale..., Contact, vol. 1, no 3: 23-25.

GENDRON, C. (1991). Développement d'un instrument d'identification des femmes violentées en milieu conjugal (Rapport de recherche). Université Laval, Les cahiers du GREMF, 43.

GHENT, W.R., N.P. DA SYLVA et M.P. FARREN (1982). La violence au foyer: directives pour la détection et l'intervention. Family violence : guidelines for recognition and management. Ottawa: Association médicale canadienne.

GIANAKOS, I. et E.E. WAGNER (1987). «Relations between Hand Test variables and the psychological characteristics and behaviors of battered women », Journal of Personality Assessment, vol. 51, $\mathrm{n}^{\circ} 2$ : 220-227.

GLEASON, W.J. (1993). "Mental Disorders in Battered Women : an Empirical Study ", Violence and Victims, vol. 8, $\mathrm{n}^{\circ} 1: 53-68$.

GOlden, G.K. et P.B. Frank (1994). «When 50-50 Isn't Fair: The Case against Couple Counseling in Domestic Abuse», Social Work, vol. 39, $\mathrm{n}^{\circ} 6: 636-637$.

HILBERMAN, E. et K. MUNSON (1977). "Sixty battered women », Victimology, vol. 2, no 3-4: 460-470.

HODGINS, S. et G. LAROUCHE (1982). «La femme violentée et les services qui lui sont offerts », Intervention, 63, 7-14.

HOUSKAMP, B.M. et D.W. FoY (1991). "The Assessment of Posttraumatic Stress Disorder in Battered Women », Journal of Interpersonal Violence, vol. $6, \mathrm{n}^{\circ} 3: 367-375$.

Hudson, W.W. et S.R. MCINTOSH (1981). "The Assessment of Spouse Abuse: Two Quantifiable Dimensions» Journal of Marriage and the Family, nov., p. 873-888.

JOHNSON, N. (1985). Marital Violence. Boston: Routledge \& Kegan.

JONES, A. et S. SCHECHTER (1992). When loves goes wrong. New York: Harper Collins.

KEMP, A., E.I. RAWLings et B.L. GReEN (1991). "Posttraumatic Stress Disorder (PTSD) in Battered Women : A Shelter Sample», Journal of Traumatic Stress, vol. 4, $\mathrm{n}^{\circ} 1: 137-148$.

KemP, A., B.L. Green, C. Hovanitz et E.I. RaWlings (1995). "Incidence and Correlates of Posttraumatic Stress Disorder in Battered Women : Shelter and Community Samples », Journal of Interpersonal Violence, vol. 10, $\mathrm{n}^{\circ} 1: 43-55$.

Kérouac, S., M.E. TAggart et J. Lescop (1986). Portrait de la santé des femmes violentées et de leurs enfants. Université de Montréal : Faculté des sciences infirmières. 
KURZ, D. et E. STARK (1988). « Not-so-benign Neglect: The Medical Response to Battering ", dans Hertsi Yllö et Michele Bograd (dir.), Feminist Perspectives on Wife Abuse, p. 249-268. Londres: Sage.

LACOMBE, M. (1990). Au grand jour. Regroupement provincial des maisons d'hébergement et de transition pour femmes victimes de violence conjugale. Montréal: Remue-ménage.

LAFRANCE, A. (1984). Éléments de dépistage et moyens pour faciliter l'expression du vécu de violence. Présentation au IX ${ }^{\mathrm{e}}$ colloque québécois sur la santé mentale. Montréal: Alternative pour Elles.

LAROuCHE, G. (1982). Protocole d'intervention en service social auprès de la clientèle des femmes violentées. Montréal: Corporation des travailleurs sociaux.

LAROUCHE, G. (1985). Guide d'intervention auprès des femmes violentées. Montréal: Corporation des travailleurs sociaux.

LAROUCHE, G. (1987). Agir contre la violence. Montréal: Pleine Lune.

LEWIS, Y.B. (1985). "The Wife Abuse Inventory: A Screening Device for the Identification of Abused Women », Social Work, vol. 30, n 1 : 32-35.

MACLEOD, L. (1980). La femme battue au Canada: un cercle vicieux. Ottawa : Conseil consultatif canadien sur la situation de la femme.

MACLEOD, L. (1987). Pour de vraies amours. Ottawa: Conseil consultatif canadien sur la situation de la femme.

MARSHALL, L.L. (1992). "Development of the Severity of Violence Against Women Scales», Journal of Family Violence, vol. 7, n 2: 103-121.

MCCAUGHRY, D. et S. CAMERON (1994). "Validation d'un outil de dépistage sur la violence conjugale», dans M. Rinfret-Raynor et S. Cantin (dir.), Violence conjugale: recherches sur la violence faite aux femmes en milieu conjugal. Boucherville: Gaëtan Morin Éditeur.

MCCAUGHRY, D. et A.D. SHARP (1991). La violence conjugale envers les femmes de l'Outaouais : validation d'un outil et de méthodes de cueillette de données. Hull : Conseil régional de la santé et des services sociaux de I'Outaouais et CLSC de la Vallée-de-la-Gatineau.

MINISTÈRE DE LA SANTÉ ET DES SERVICES SOCIAUX (1987). Une politique d'aide aux femmes violentées. Québec.

Ministère DE LA SANTÉ ET DES SERVICES SOCIAux (1992). La politique de la santé et du bien-être. Québec.

ORDRE DES INFIRMIÈRES ET INFIRMIERS DU QUÉBEC (1987). Écouter le langage des maux: la violence conjugale. Intervention infirmière auprès des femmes. Montréal: OIIQ.

Orem, D.E. (1985). Nursing Concepts of Practice. Toronto: McGraw-Hill.

PÂQUET-DEEHY, A.M. RINFRET-RAYNOR et G. LAROUCHE (1989). Apprendre à intervenir auprès des femmes violentées : une perspective féministe. Rapport de recherche sur une expérience de formation. Université de Montréal: École de service social.

Post, R.D., A.B. Willett, R.D. Franks, R.M. HOUSE, S.M. BACK et M.P. WEISSBERG (1980). "A preliminary report on the prevalence of domestic violence among psychiatric impatients", American Journal of Psychiatry, 137: 974-975.

PRANSKY, J. (1991). Prevention : The Critical Need. Springfield: Burrel. 
RAYMOND, M. et S. ChARBONNEAu (1984). À tous coups : ressources contre la violence. Montréal : Remue-ménage.

Regroupement des CLSC du MONTRÉAL métropolitain (1990). Guide d'animation sur le dépistage de la violence conjugale. Montréal.

ReGroupement des CLSC Du MONTRÉAl métropolitAin (1990). Guide de dépistage de la violence conjugale. Montréal.

REGROUPEMENT PROVINCIAL DES MAISONS D'HÉBERGEMENT ET DE TRANSITION POUR FEMMES VICTIMES DE VIOLENCE CONJUGALE (1990). La violence conjugale, c'est quoi au juste? Montréal: Regroupement provincial des maisons d'hébergement et de transition pour femmes victimes de violence conjugale.

Rinfret-Raynor, M., A. PÂquet-Deehy, G. Larouche et S. CANTin (1989). Intervenir auprès des femmes violentées : évaluation de l'efficacité d'un modèle féministe. Méthodologie de la recherche et caractéristiques des participantes. Rapport de recherche $n^{\circ} 1$. Université de Montréal: École de service social.

Rinfret-Raynor, M., A. PÂQuet-Deehy, G. Larouche et S. CANTin (1991). Intervenir auprès des femmes violentées : évaluation de l'efficacité d'un modèle féministe. Présentation et analyse des résultats. Rapport de recherche $n^{\circ}$ 2. Université de Montréal: École de service social.

Rinfret-Raynor, M., A. PÂquet-Deehy, G. Larouche et S. CANTin (1992). Intervenir auprès des femmes violentées : évaluation de l'efficacité d'un modèle féministe. Montréal: Saint-Martin.

RoDenburG, F.A. et J.W. FAntuzzo (1993). "The Measure of Wife Abuse: Steps toward the Development of a Comprehensive Assessment Technique », Journal of Family Violence, vol. 8, no 3 : 203-228.

RODGERS, K. (1994). "Résultats d'une enquête nationale sur I'agression contre la conjointe », Juristat, Bulletin de service du Centre canadien de la statistique juridique, Statistique Canada, vol. 14, $\mathrm{n}^{\circ}$ 9: 1-22.

Rose, S.M., G. Peabody et B. Stratigeas (1991). "Responding to Hidden Abuse: A Role for Social Work in Reforming Mental Health Systems », Social Work, vol. 36, $\mathrm{n}^{\circ} 5: 408-413$.

ROSEWATER, L.B. (1985). "Schizophrenic, borderline, or battered?», dans L. B. Rosewater et L.E.A. Walker (dir.), Handbook of feminist therapy: Women's issues on psychotherapy (p. 215-222). New York: Springer.

SINCLAIR, D. (1985). Pour comprendre le problème des femmes battues : Guide de formation pour les conseillers et les intervenants. Toronto: Ministère des Services sociaux et communautaires, programme de lutte contre la violence au foyer.

StARK, E., A. FlitCraft et W. Frazier (1979). "Medicine and Patriarcal violence: the social construction of a private event», International Journal of Health Services, 9: 461-493.

StArk, E., A. Flitcraft, D. ZuCKerman, A. Grey, J. Robinson et W. FraZier (1981). Wife abuse in the medical setting : an introduction for healthpersonnel. Rockville: National Clearinghouse on Domestic Violence.

STATISTIQUE CANADA (1993). "L'enquête sur la violence envers les femmes », Le Quotidien, 18 novembre, p. 1-10.

Straus, M.A., R. Gelles et S. Steinmetz (1980). Behind closed doors. Violence in the American family. New York: Anchor Press. 
TURGEON, J. (1992). La prévention de la violence conjugale: Examen général de doctorat. Montréal: Université de Montréal, Département de psychologie.

TURGEON, J. (1995). Les conséquences de la violence familiale : effets combinés des multiples victimisations. Texte inédit d'une conférence présentée au Vingtième congrès de la Fédération des CLSC du Québec, Montréal.

TURNER, S.F. et C.H. SHAPIRO (1986). "Battered women: Mourning the death of a relationship », Social Work, vol. 31, no $5: 372-376$.

WALKER, L.E.A. (1979). The battered woman. New York: Harper \& Row.

WALKER, L.E.A. (1983). "The battered woman syndrome», dans D. Finkelhor, R.J. Gelles, G.T. Hotaling, M.A. Straus (dir.), The Dark Side of Families. Beverly Hills: Sage.

WALKER, L.E.A. (1984). The battered woman syndrome. New York: Springer.

WALKER, L.E.A. (1985). "Psychological impact of the criminalization of domestic violence on victims », Victimology: An International Journal, $10: 281-300$.

Walus Wigle, J. et J.R. Meloy (1988). "Battered woman syndrome as a criminal defense », Journal of Psychiatry and Law, vol. 16, $\mathrm{n}^{\circ} 3$ : 389-404.

WARREN, R.L., S.M. ROSE et A.F. BURGUNDER (1974). The structure of urban reform. Lexington: Heath.

WARSHAW, C. (1989). "Limitations of the medical model in the care of battered women », Gender and Society, vol. 3, no 4 : 506-517. 